DOI: $10.23857 /$ dc.v5i3.942

Ciencias técnicas y aplicadas

Artículo de revisión

\title{
Modelo de comercialización para las potencialidades productivas de los derivados de la caña de azúcar
}

\section{Marketing model for the productive potential of sugar cane derivatives}

\section{Modelo de comercialização para as potencialidades produtivas dos derivados da caña de azúcar}

\author{
Xavier Enrique Soledispa-Rodríguez ${ }^{\mathrm{I}}$ \\ xavier.soledispa@unesum.edu.ec \\ Carlos Artemidoro Zea-Barahona II \\ carlos.zea@unesum.edu.ec \\ Antonio Eduardo Osejos-Vásquez ${ }^{\mathrm{III}}$ \\ antonio.osejos@unesum.edu.ec \\ Hernán Patricio Delgado-Solís IV \\ hernanpatricio.del@gmail.com
}

Recibido: 25 de abril de 2019 *Aceptado: 27 de junio de 2019 * Publicado: 05 de julio de 2019

I. Especialista en Consultoría de Proyectos, Economista, Profesor Titular Principal Carrera Administración de Empresas, Facultad de Ciencias Económicas, Universidad Estatal del Sur de Manabí, Jipijapa, Manabí, Ecuador.

II. Magíster en Docencia Universitaria e Investigación Educativa, Economista, Profesor Titular Principal Carrera Administración de Empresas, Facultad de Ciencias Económicas de la Universidad Estatal del Sur de Manabí, Jipijapa, Manabí, Ecuador.

III. Magíster en Finanzas y Comercio Internacional, Profesor Ocasional Tiempo Completo de la Universidad Estatal del Sur de Manabí, Jipijapa, Manabí, Ecuador.

IV. Magíster en Contabilidad y Auditoría, Profesor Ocasional Tiempo Completo de la Universidad Estatal del Sur de Manabí, Jipijapa, Manabí, Ecuador. 


\title{
Resumen
}

El objeto de estudio que permitió la realización de la primera fase de la presente investigación se basó en identificar el sistema de control de calidad y determinar los canales de distribución que tienen implementado los productores del sitio San Carlos del cantón Jipijapa, en el procesamiento de los derivados de la caña de azúcar. se debe implementar un sistema de control de calidad, no existe desde la vestimenta adecuada de los trabajadores hasta el almacenamiento de los productos terminados, un sistema que garantice la conservación del aguardiente, raspadura en bloque, alfeñique, la comercialización la realizan de forma directa con intermediarios y consumidores finales.

Palabras clave: Sistema de control; calidad; almacenamiento; aguardiente; alfeñique; raspadura.

\begin{abstract}
The object of the study that enabled the realization of the first phase of the present investigation is based on identifying the quality control system and determining the distribution channels that have been implemented by the producers of the San Carlos Canton Jipijapa site, in the process of processing them. sugar cane derivatives. It is necessary to implement a quality control system, since there is no proper garment of the workers there is the storage of finished products, a system that ensures the preservation of the brand, scraping in the lock, letting, the commercialization of the direct accomplishment with intermediaries and final consumers.
\end{abstract}

Keywords: Control system; quality; storage; brandy; alpine; scratching.

\section{Resumo}

O objeto de estudo que permitiu a realização da primeira fase da investigação se identificou como o sistema de controle de qualidade e de determinação dos canais de distribuição que implementaram os produtos do setor San Carlos do cantão de Jipijapa, no processo de derivados

\footnotetext{
392 Vol. 5, núm. 3, julio, 2019, pp.391-414 Enrique Soledispa-Rodríguez, Carlos Artemidoro Zea Barahona, Antonio Eduardo Osejos Vásquez, Hernán Patricio Delgado Solís
} 
da cana de azúcar. A fim de implementar um sistema de controle de qualidade, não existe uma vestimenta a adecuada de los trabajadores hasta el almacenamiento de los productos terminados, un sistema que garante a conservação da bebida, raspadura em blocos, alfeñique, la comercialización la realizan de forma directa con intermediários e consumidores finais.

Palavras chaves: Sistema de controle; calidad; almacenamiento; aguardente; alfeñique; raspadura.

\section{Introducción}

La caña de azúcar es considerada uno de los principales productos de producción en América Latina, para Ecuador su representación tiene su importancia, aunque en menor grado. Los datos del Banco Central, nos informa, que: la producción de la caña de azúcar contribuye con el 1,4\% al PIB nacional y genera más de 30.000 empleos directos y 80.000 indirectos sobre todo en la época seca de su cosecha (de julio a diciembre).

En el área de influencia del sitio San Carlos existen agrupaciones familiares de productores que están trabajando en el sector agrícola y esto hay que aprovecharlo para crear más emprendimientos en beneficios de la comunidad.

La producción de caña de azúcar en este sitio la desarrollan pequeños productores, en algunos casos para la industrialización del aguardiente y producción de miel negra para elaborar la panela o raspadura y el alfeñique. Trabajan a través del comité de productores que cuentan con una débil organización institucional y empresarial.

El mayor problema que enfrentan es la deficiente comercialización de los productos derivados de la caña de azúcar, cuyas causas principales son: La débil organización, Deficientes recursos operativos, Poco entendimiento entre los propios integrantes, una visión empresarial muy limitada, No hay organización para la producción, transformación y comercialización de la caña de azúcar, Los precios de la panela son bajos no cubren los gastos de producción (la panela de 3 libras se

\footnotetext{
393 Vol. 5, núm. 3, julio, 2019, pp.391-414 Enrique Soledispa-Rodríguez, Carlos Artemidoro Zea Barahona, Antonio Eduardo Osejos Vásquez, Hernán Patricio Delgado Solís
} 
vende a 1 dólar, en escases se vende a 1,50 US \$), No hay capital que permita acopiar la panela para vender en el invierno, Excesivo control del Estado en la producción y comercialización de aguardiente y Los productores que antes producían aguardiente, ahora están produciendo panela lo que incrementa la oferta en el mercado disminuyendo los precios.

Entre los Problemas Comunes tenemos: Falta de apoyo técnico, y tecnológico de instituciones de manera articulada, No existen procesos de capacitación técnica, administrativa y contable, Dificultades para acceder al crédito público, Deficiente sostenibilidad en la organización y Falta de campañas de forestación con fines productivos.

Por tal motivo se busca conjugar la información necesaria para entender su funcionamiento, problemática y necesidades, generando un documento base para integrar el Modelo de Comercialización dentro de la cadena con un enfoque participativo entre los diferentes agentes que intervienen en su desarrollo.

El impacto científico no es muy notorio, ya que el proceso para obtener la materia prima para elaborar los derivados de la caña de azúcar es rústico, debe implementarse una tecnología que permita obtener un producto de calidad. Los residuos de la caña molida son agrupados en un determinado lugar y luego son quemados, lo que causa un problema al medio ambiente, no se está aplicando una alternativa para aprovechar estos desperdicios y asignarle un valor agregado (solamente una persona está empleando los mismo para hacer abono orgánico). Socialmente no existe una responsabilidad de aplicar alguna alternativa para mejorar las condiciones de la comunidad, no se ha creado ningún proyecto en base a la responsabilidad social que tienen las empresas para con la sociedad. Económicamente el impacto se lo visualizara con la corrección de los problemas detectados, ya que estos conllevan a mejorar todos los procesos y responsabilidad que tiene toda inversión.

\footnotetext{
394 Vol. 5, núm. 3, julio, 2019, pp.391-414 Enrique Soledispa-Rodríguez, Carlos Artemidoro Zea Barahona, Antonio Eduardo Osejos Vásquez, Hernán Patricio Delgado Solís
} 
Para esto se planteó como objetivo general "Diseñar un modelo de comercialización para la potenciación de la productividad de los derivados de la caña de azúcar y mejorar la economía de los productores", con el cumplimiento de las siguientes actividades:

Identificar qué sistema de control de calidad tienen implementado los productores en el procesamiento de los derivados de la caña de azúcar.

Determinar los canales de distribución de los productos derivados de la caña de azúcar de los productores del sitio San Carlos del cantón Jipijapa. En su primera fase-Para su segunda fse las actividades son:

Establecer capacitaciones para la elaboración del modelo de comercialización de los productos derivados de la caña de azúcar, con una cultura de responsabilidad social corporativa entre proveedores y productores, que propicien el crecimiento de la iniciativa empresarial.

Elaborar y validar un modelo de comercialización para la distribución de los productos derivados de la caña de azúcar.

\section{Materiales y Métodos}

La presente investigación fue de tipo no experimental y se desarrolló bajo un enfoque cuantitativo, cualitativo, bibliográfica, documental, basado en los métodos: descriptivo, inductivo, deductivo y analítico, apoyado con las técnicas de observación, entrevista y encuestas.

\section{Resultados}

Aspectos generales de las microempresas de los derivados de caña de azúcar del sitio San Carlos.

El sitio San Carlos se caracteriza por ser productor de caña de azúcar donde gran parte de su población se dedica a esta actividad.

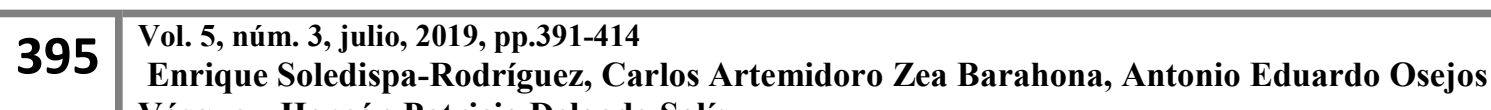
Vásquez, Hernán Patricio Delgado Solís
} 
La caña de azúcar es una planta de la cual se obtiene una diversidad de productos (Aguardiente, Panela, Alfeñique, punto), los mismos que mediante su comercialización se han convertido en el sustento familiar de esta población, contribuyendo al desarrollo social, económico, productivo y empresarial.

En este sitio existen 6 microempresas que se dedican a darle valor agregado a este producto, las mismas que cuentan con las siguientes características

Cuadro 1: Datos generales de la microempresa "Las delicias de caña de azúcar"

\begin{tabular}{|l|l|}
\hline Microempresa & Las delicias de la caña de azúcar \\
\hline Propietario & Luis Alberto Zorrilla Tóala \\
\hline Años de funcionamiento & 14 Años \\
\hline Área de producción (m²) & $90 \mathrm{~m}^{2}$ \\
\hline Productos que elabora & Panela, melcocha con maní \\
\hline Producción mensual & 85 litros día \\
\hline Empleados (Zafra) & Dos \\
\hline Empleados (producción) & Seis \\
\hline Permisos de funcionamiento & Cuenta con permiso de funcionamiento \\
\hline Ingresos mensuales & \\
\hline
\end{tabular}

La materia prima principal para producir los derivados de la caña de azúcar proviene de su propio cultivo, con un área de producción de 2 hectáreas.

La transformación de la caña de azúcar en jugo se la realiza en los meses de octubre a diciembre donde trabajan seis personas en zafra (sacar la caña de azúcar de la plantación) y dos en la transformación del producto.

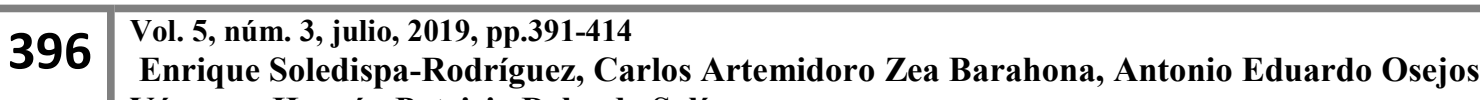
Vásquez, Hernán Patricio Delgado Solís
} 
Esta microempresa cuenta con los permisos necesarios para funcionamiento, sin embargo, sus productos no cuentan con ninguna marca que lo represente en el mercado para su comercialización, el producto es distribuido por canales directos e indirectos, generando ingresos mensuales en un promedio de 400 dólares.

Esta organización se encuentra asociada en ASOSAGRA presidida por el señor Ramón Zorrilla la misma que cuenta con 22 socios, de la cual se encuentran inactivo por no percibir ningún beneficio.

Cuadro 2: Datos generales de la microempresa "San Carlos"

\begin{tabular}{|l|l|}
\hline Microempresa & San Carlos \\
\hline Propietario & Gloria Yoza de Zorrilla \\
\hline Años de funcionamiento & 18 años \\
\hline Área de producción (m²) & $95 \mathrm{~m}^{2}$ \\
\hline Productos que elabora & Panela, melcocha con maní, Punto \\
\hline Producción mensual & 120 litros día, 400 panela, 400 melcocha, punto 150 \\
\hline Empleados (Zafra) & Dos \\
\hline Empleados (producción) & Cuatro \\
\hline Permisos de funcionamiento & Cuenta con permiso de funcionamiento \\
\hline Ingresos mensuales & \\
\hline
\end{tabular}

Esta microempresa se dedica a la elaboración de aguardiente, cuenta con un área de cultivo de caña de azúcar orgánica de 20 hectáreas, donde trabajan en temporada de zafra seis personas, mientras que durante la producción de los derivados sólo hay dos trabajadores.

Su producción es de 1201ts./día, el mismo que se comercializa a un valor de \$2,00 el litro, al igual que los productores anteriores, a pesar de encontrarse asociado a la organización ASOSAGRA, no participa de la misma ni recibe desde hace mucho tiempo ningún beneficio, tiene permiso de

\footnotetext{
\begin{tabular}{l|l} 
Vol. 5, núm. 3, julio, 2019, pp.391-414 \\
Enrique Soledispa-Rodríguez, Carlos Artemidoro Zea Barahona, Antonio Eduardo Osejos
\end{tabular} Vásquez, Hernán Patricio Delgado Solís
} 
funcionamiento, pero no cuenta con una marca y nombre de su producto para su comercialización, lo vende a intermediarios (minoristas).

Cuadro 3: Datos generales de la microempresa

\begin{tabular}{|l|l|}
\hline Microempresa & S.N \\
\hline Propietario & José Baque \\
\hline Años de funcionamiento & 10 años \\
\hline Área de producción (m²) & $75 \mathrm{~m}^{2}$ \\
\hline Productos que elabora & Alfeñique, raspadura \\
\hline Producción mensual & 350 Alfeñique, 400 raspadura \\
\hline Empleados (Zafra) & 4 \\
\hline Empleados (producción) & 2 \\
\hline Permisos de funcionamiento & Cuenta con permiso de funcionamiento \\
\hline Ingresos mensuales & \\
\hline
\end{tabular}

Esta microempresa se dedica a la elaboración de alfeñique y raspadura cuenta con un área de cultivo de caña de azúcar orgánica de 3 hectáreas, donde trabajan en temporada de zafra cuatro personas, mientras que durante la producción de los derivados sólo trabajan dos.

Cuadro 4: Datos generales de la microempresa "El Amparo"

\begin{tabular}{|l|l|}
\hline Microempresa & "El Amparo” \\
\hline Propietario & Guadalupe María Zorrilla Tóala \\
\hline Años de funcionamiento & Seis años \\
\hline Área de producción $\left.\mathbf{( m}^{\mathbf{2}}\right)$ & $80 \mathrm{~m}^{2}$ \\
\hline Productos que elabora & Aguardiente \\
\hline
\end{tabular}

\footnotetext{
398 Vol. 5, núm. 3, julio, 2019, pp.391-414

Enrique Soledispa-Rodríguez, Carlos Artemidoro Zea Barahona, Antonio Eduardo Osejos Vásquez, Hernán Patricio Delgado Solís
} 


\begin{tabular}{|l|l|}
\hline Producción & 180 lts./día \\
\hline Empleados (Zafra) & Seis \\
\hline Empleados (Producción) & Dos \\
\hline Permisos de funcionamiento & Cuenta con permiso de funcionamiento \\
\hline Ingresos mensuales & \\
\hline
\end{tabular}

Este productor se dedica a la elaboración de aguardiente y está incursionando en la elaboración de abono del bagazo de la caña de azúcar, cuenta con un área de cultivo de 6 hectáreas.

En temporada de zafra trabajan seis personas, y en el proceso de trasformación de la materia prima solamente dos.

Su producción es de 180 lts./día en los meses octubre a noviembre, este producto es comercializado a un valor de $\$ 2,00$ el litro, al igual que el productor anterior, a pesar de encontrarse asociado a la organización ASOSAGRA, no participa de la misma ni recibe desde hace mucho tiempo ningún beneficio. Tiene permiso para su funcionamiento, pero no cuenta con una marca y nombre de su producto final para su comercialización, lo vende a intermediarios (minoristas).

Cuadro 5: Datos generales de la microempresa "San Carlos"

\begin{tabular}{|l|l|}
\hline Microempresa & San Carlos \\
\hline Propietario & Gloria Vivar Gómez \\
\hline Años de funcionamiento & 30 Años \\
\hline Área de producción $\mathbf{( m}^{\mathbf{2}}$ ) & $70 \mathrm{~m}^{2}$ \\
\hline Productos que elabora & Aguardiente \\
\hline Producción & 120 lts./día \\
\hline Empleados (Zafra) & Cinco personas \\
\hline Empleados (Producción) & Cuatro \\
\hline
\end{tabular}

399 Vol. 5, núm. 3, julio, 2019, pp.391-414

Enrique Soledispa-Rodríguez, Carlos Artemidoro Zea Barahona, Antonio Eduardo Osejos Vásquez, Hernán Patricio Delgado Solís 


\begin{tabular}{|l|l|}
\hline Permisos de funcionamiento & Cuenta con permiso de funcionamiento \\
\hline Ingresos mensuales & \\
\hline
\end{tabular}

Esta microempresa se dedica a la elaboración de aguardiente, cuenta con un área de cultivo de caña de azúcar orgánica de 3 hectáreas, donde trabajan en temporada de zafra cinco personas, mientras que durante la producción de los derivados sólo trabajan 4, los mismos son integrantes de la familia y trabajan los terrenos y son parte de la repartición de utilidades con la dueña.

Su producción es de 120 lts./día, el mismo que se comercializa a un valor de $\$ 2,00$ el litro, la familia a cargo está compuesta por 4 personas que son sus beneficiarias, y al igual que los productores anteriores, a pesar de encontrarse asociado a la organización ASOSAGRA, no participa de la misma ni recibe desde hace mucho tiempo ningún beneficio, Tiene permiso de funcionamiento, pero no cuenta con una marca y nombre de su producto para su comercialización, lo vende a intermediarios (minoristas).

Cuadro 6: Datos generales de la microempresa "San José"

\begin{tabular}{|l|l|}
\hline Microempresa & "San José" \\
\hline Propietario & José Zorrilla Tóala \\
\hline Años de funcionamiento & 6 Años \\
\hline Área de producción $\left.\mathbf{( m}^{\mathbf{2}}\right)$ & $92 \mathrm{~m}^{2}$ \\
\hline Productos que elabora & Aguardiente \\
\hline Producción & 80 lts /días \\
\hline & \\
\hline Empleados (Zafra) & Cuatro \\
\hline Empleados (Producción) & Dos \\
\hline Permisos de funcionamiento & Cuenta con permiso de funcionamiento \\
\hline
\end{tabular}

\footnotetext{
\begin{tabular}{l|l}
\hline Vol. 5, núm. 3, julio, 2019, pp.391-414 \\
Enrique Soledispa-Rodríguez, Carlos Artemidoro Zea Barahona, Antonio Eduardo Osejos
\end{tabular} Vásquez, Hernán Patricio Delgado Solís
} 


\section{Ingresos mensuales}

Este cañicultor se dedica a la elaboración de aguardiente, cuenta con un área de cultivo de caña de azúcar orgánica de 3 hectáreas, donde trabajan durante todo el año 2 personas, abasteciendo constantemente a sus dueños del producto para su expendio en la ciudad de Jipijapa.

Su producción es de 80 lts./día, el mismo que se comercializa a un valor de $\$ 3,50$ el litro en variedades como; puro, curado (sabor) y cocteles, al igual que los productores anteriores, a pesar de encontrarse asociado a la organización ASOSAGRA, no participa de la misma ni recibe desde hace mucho tiempo ningún beneficio. Tiene certificado sanitario, y una marca y nombre para su comercialización ("La Libra"), lo que le permite expender sus productos de manera directa al consumidor final (canal de distribución corto), aumentando su rentabilidad.

\section{Infraestructura del área de producción}

La infraestructura de estas microempresas fue evaluada mediante una ficha de observación, conformada por diferentes elementos, obteniendo los siguientes resultados:

\section{1.- Especificación técnica del área de la empresa}

El área promedio donde funcionan estas empresas es de tres hectáreas donde su mayor parte es ocupada en el cultivo de la caña de azúcar, y alrededor de unos $80 \mathrm{~m}^{2}$ en el área de producción y almacenamiento, $40 \mathrm{~m}^{2}$ para desechos de la caña azúcar y $50 \mathrm{~m}^{2}$ en la vivienda de los propietarios, no se cuenta las siguientes áreas necesarias para su funcionamiento:

- Área Administrativa

- Área de control de calidad

- Área Verde

\section{2.- Especificación técnica de la estructura de la edificación}

\footnotetext{
\begin{tabular}{l|l}
\hline & Vol. 5, núm. 3, julio, 2019, pp.391-414 \\
Enrique Soledispa-Rodríguez, Carlos Artemidoro Zea Barahona, Antonio Eduardo Osejos
\end{tabular} Vásquez, Hernán Patricio Delgado Solís
} 
Estas microempresas solo cuentan con un área de producción y almacenamiento el mismo que es un galpón, su estructura está construida en tres de ellas con madera y caña, las dos con metal y una de hormigón, el techo es de metal, el piso de hormigón, no se encuentra cubierto de los lados por motivo de ventilación.

\section{3.- Especificación técnica del sistema eléctrico hidráulica y sanitaria.}

En lo corresponde a las instalaciones eléctricas se analizaron los siguientes puntos: tableros electrónicos, disyuntor, termo magnético, conductores de diferentes colores, toma corrientes, interruptores para iluminación, artefactos luminosos adecuados, puesta a tierra.

\section{a.-Tableros electrónicos}

EL tablero electrónico es uno de los componentes principales de una instalación eléctrica, en él se protegen cada uno de los distintos circuitos en los que se divide la instalación a través de fusibles, protecciones magnetotérmicas y diferenciales.

\section{b.- Disyuntores termomagnéticos}

El disyuntor termomagnético o llave térmica, es un dispositivo capaz de interrumpir la corriente eléctrica de un circuito cuando ésta sobrepasa ciertos valores máximos.

\section{c.- Disyuntor termo magnéticos}

Es un dispositivo capaz de interrumpir la corriente eléctrica de un circuito cuando ésta sobrepasa ciertos valores máximos.

\section{d.- Conductores eléctricos}

Un conductor eléctrico es un material que ofrece poca resistencia al movimiento de la carga eléctrica. Sus átomos se caracterizan por tener pocos electrones en su capa de valencia, por lo que no se necesita mucha energía para que estos salten de un átomo a otro.

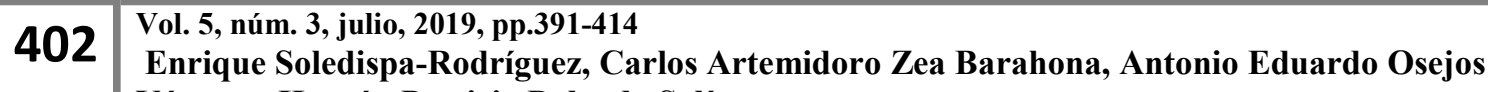
Vásquez, Hernán Patricio Delgado Solís
} 


\section{e.- Tomacorrientes}

Un enchufe está formado por dos elementos: clavijas y tomacorriente (o toma de corriente), que se conectan uno al otro para establecer una conexión que permita el paso de la corriente eléctrica.

\section{f.-Interruptores}

Un interruptor es un dispositivo eléctrico que nos permite realizar una función de on/off desde un mando. Su funcionamiento consiste en dejar pasar o no la corriente en un circuito eléctrico. Por ello, su función principal es el encendido y apagado de una luz.

\section{g.- Puesta a tierra}

Es la conexión de las superficies conductoras expuestas (gabinetes metálicos) a algún punto no energizado; comúnmente es la tierra sobre la que se posa la construcción, de allí el nombre. Al sistema de uno o varios electrodos que proveen la conexión a tierra se le llama «toma de tierra». Las puestas a tierra se emplean en las instalaciones eléctricas como una medida de seguridad.

De acuerdo a cada una de los elementos expuesto para evaluar el funcionamiento adecuado del sistema eléctrico de la empresa se encontraron los siguientes resultados:

Ninguna de las microempresas cuenta con un adecuado sistema eléctrico ya que no cumplen con todos los elementos que se han establecido para su evaluación.

En lo que respecta al uso de tableros electrónicos ninguna de las microempresas utiliza este equipo para el controlar y proteger cada uno de los distintos circuitos en los que se divide la instalación a través de fusibles. En lo que corresponde al uso de disyuntor termomagnético no es utilizado sin embargo se utilizan Breakers para interrumpir la corriente eléctrica de un circuito cuando ésta sobrepasa ciertos valores máximos. En el cableado en algunas de estas microempresas necesitan ser reemplazados, como también los interruptores y toma corrientes tampoco se cuenta con la conexión puesta a tierra la cual es muy importante para cualquier descarga que se presente.

\footnotetext{
403 Vol. 5, núm. 3, julio, 2019, pp.391-414

Enrique Soledispa-Rodríguez, Carlos Artemidoro Zea Barahona, Antonio Eduardo Osejos Vásquez, Hernán Patricio Delgado Solís
} 
En lo que respecta al abastecimiento de agua esta proviene de los pozos existentes en la comunidad, la cual es almacenada en cisternas y reservorios de plásticos PVC, de donde se distribuye para diferentes áreas mediante tuberías de PVC.

\section{4.- Señalizaciones de seguridad}

La señalización de seguridad es uno de los elementos más importantes para garantizar y preservar la seguridad de todos los trabajadores y personas que se encuentren en el ambiente de trabajo, sirviendo para alertar y orientar a los profesionales acerca de los riesgos existentes en la empresa $\mathrm{y}$ ofrecer orientaciones a las personas que frecuentan el local.

Para la evaluación de la señalización de seguridad se consideraron los siguientes elementos:

- Señales de prohibición

- Señales de obligación para la seguridad de los trabajadores

- Señales de advertencia sobre las instalaciones

- Señales de auxilio necesarias en el lugar de trabajo

- Señales luminosas

Los resultados de esta evaluación es que ninguna de estas microempresas cuenta con este tipo de señalización.

\section{Sistema de control de calidad}

El término control de calidad se refiere "a un sistema organizativo por medio del cual la producción se ajustará a unos parámetros específicos que definen la calidad del producto o servicio” (...) (Lester, Enrick, \& Mottley, 1989)

"Por control de calidad, en su acepción moderna, se entiende un sistema de procedimientos para producir en forma económica bienes y servicios que satisfagan los requerimientos del consumidor”. (Gutiérrez, 1989)

\footnotetext{
404 Vol. 5, núm. 3, julio, 2019, pp.391-414

Enrique Soledispa-Rodríguez, Carlos Artemidoro Zea Barahona, Antonio Eduardo Osejos Vásquez, Hernán Patricio Delgado Solís
} 
"La nueva filosofía administrativa supone, pues, que la calidad es objeto de planificación y que se consigue como resultado de un mejoramiento del proceso" (Ibídem)

El control de calidad abarca dos aspectos principales. El primero se refiere a la capacidad y a la medición real de las características de la calidad que son significativas en cuanto al comportamiento del producto. El segundo se refiere a proporcionar los caminos que conducen a la acción correctora en todos aquellos casos en los que las mediciones de la calidad indican un comportamiento significativamente fuera del estándar -en términos de materiales de recepción, fabricación de componentes o montaje final (Lester, Enrick, \& Mottley, 1989).

El proceso de control de calidad tiene lugar dentro del marco de la aplicación de sistemas. El objetivo del sistema de control de calidad es generalmente la consecución de unos niveles particulares de calidad, tal como se indican en las especificaciones y tolerancias. Las características importantes de estas especificaciones incluyen la descripción exacta del producto, los límites claramente definidos de varias características, los estándares de las medidas directas (tales como las dimensiones) o medidas indirectas (tales como contenido de humedad, deducido a partir de las lecturas de resistencia eléctrica), y la diferenciación entre características de calidad mayores o críticas y los defectos menores o menos importantes. La vía para la consecución del objetivo del sistema de control de calidad pasa a través del equipo de producción, el personal, y los servicios de procesamiento, operaciones y similares. El seguimiento es el paso final, por medio del cual se garantiza el control de calidad. (Ibídem)

\section{Programa de control de calidad}

De acuerdo a (Lester, Enrick, Mottley (Jr), \& Medina, 1989). Un programa es la estructura global que sirve para la definición y el análisis de los objetivos del sistema. Abarca los siguientes pasos:

\section{- Primer paso}

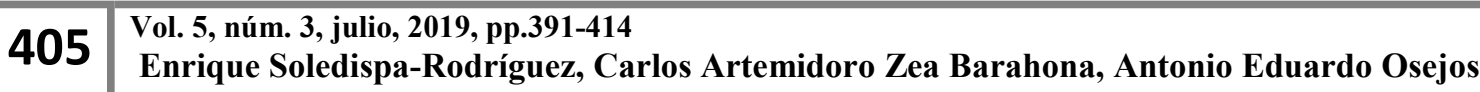
Vásquez, Hernán Patricio Delgado Solís
} 
Revisión de las especificaciones, para asegurarse de que las necesidades y deseos del cliente serán satisfechos. Con este fin se necesitan especificaciones exactas y precisas.

\section{- Segundo paso A:}

Diagrama de flujo que documenten las operaciones individuales y los productos a lo largo de los distintos procesos de producción. Cuando surgen problemas de calidad, estos procesos deben ser rastreados para buscas mejoras eficaces.

\section{- Segundo paso B:}

Se describen los procedimientos operativos para cada una de las operaciones del diagrama de flujo. Nuevamente, el objetivo es disponer de información que sea útil cuando se comprueban las operaciones del comportamiento de la calidad. Cuando hay de por medio requisitos rigurosos las especificaciones del proceso sustituyen a las simples descripciones del proceso.

\section{- Tercer paso:}

Las auditorías de calidad, por medio de las cuales se mide y registra la calidad, garantizan que se satisfacen las especificaciones y tolerancias. Las auditorías de calidad deben estar basadas en planes de inspección y frecuencias de ensayo, conocidas como planes de calidad.

\section{- Cuarto paso:}

Periódicamente se analiza el comportamiento de la calidad a partir de los historiales acumulados de la calidad. De este modo, se identifican los problemas de calidad de larga duración para ser corregidos. Estos historiales, junto con otros datos acumulados proporcionan el feedback para producción, indicando los lugares donde hace falta una acción correctora. (Lester, Enrick, \& Mottley, 1989)

\footnotetext{
406 Vol. 5, núm. 3, julio, 2019, pp.391-414 Enrique Soledispa-Rodríguez, Carlos Artemidoro Zea Barahona, Antonio Eduardo Osejos Vásquez, Hernán Patricio Delgado Solís
} 
En resumen, el primer y segundo paso definen lo que el proceso trata de lograr, el tercer paso mide los resultados obtenidos, y el cuarto paso suministra la información para mejorar el comportamiento a largo plazo.

De acuerdo a lo establecido se recabo información de la cual se obtuvo lo siguiente:

Los propietarios de estas microempresas indicaron que ellos planifican su producción la cual comienza en el mes de octubre y termina en diciembre, indicaron también que el sistema de producción que emplean es adecuado y que constantemente evalúan la calidad de su producto ya que consideran que es de mucha importancia este proceso, ya que este comienza desde la materia prima, proceso de fabricación, envasado y presentación del producto, como también un adecuado almacenamiento.

También indicaron que el control de calidad le permite identificar problemas en el producto y mejorarlos, para así cumplir con la exigencia de los consumidores y poder posicionarse en el mercado.

Sin embargo, al recopilar información en el área de producción se detectó lo siguiente:

- Se carece de medios de control en los procesos

- No se realizan estudios para saber si el cliente está a gusto con el producto

- Estima los costos con base a la experiencia e intuición

- No se lleva a cabo ningún tipo de registro

- No se lleva a cabo ningún inventario

- Carece de normas de seguridad

- La maquinaria es suficiente pero no adecuada o bien es adecuada pero no suficiente

- La empresa no dispone de un laboratorio interno

- No existen procedimientos/instrucciones escritos referentes a la actividad de control durante las fases de producción o diseño, y para la aceptación final

\footnotetext{
407 Vol. 5, núm. 3, julio, 2019, pp.391-414

Enrique Soledispa-Rodríguez, Carlos Artemidoro Zea Barahona, Antonio Eduardo Osejos Vásquez, Hernán Patricio Delgado Solís
} 
- $\quad$ No existe un procedimiento/instrucción escrito para la gestión y el control de la configuración del producto.

Mediante la siguiente ficha se evaluaron los siguientes aspectos obteniendo los siguientes resultados.

\section{Cuadro 7: Ficha de observación}

\begin{tabular}{|l|c|c|}
\hline \multicolumn{1}{|c|}{ ASPECTOS A EVALUAR } & SÍ & NO \\
\hline 1.- MATERIA PRIMA & & \\
\hline Es seleccionada & $\mathbf{5}$ & $\mathbf{6}$ \\
\hline El lugar de almacenamiento es adecuado & & $\mathbf{6}$ \\
\hline El lugar de almacenamiento se encuentra al aire libre. & & \\
\hline El lugar de almacenamiento es rustico. & $\mathbf{6}$ & \\
\hline 2.- MAQUINARIA & & \\
\hline Es moderna & $\mathbf{6}$ & \\
\hline Es rustica & & \\
\hline Es moderna y rustica & & \\
\hline Es digital & $\mathbf{6}$ & \\
\hline Es mecánica & & \\
\hline Es eléctrica & $\mathbf{6}$ & \\
\hline Es a gasolina & & \\
\hline Es a diésel & & \\
\hline Es manual & & \\
\hline 3.- ENVASADO & & \\
\hline Es mecánico & & \\
\hline Es electrónico & & \\
\hline
\end{tabular}




\begin{tabular}{|l|c|c|}
\hline Es manual & $\mathbf{6}$ & \\
\hline El material posee normas de calidad & & $\mathbf{6}$ \\
\hline Tiene un nombre & $\mathbf{2}$ & $\mathbf{4}$ \\
\hline Tiene un logotipo & $\mathbf{2}$ & $\mathbf{4}$ \\
\hline Tiene registro sanitario & $\mathbf{6}$ & \\
\hline 4.- ALMACENAMIENTO (Producto final) & & \\
\hline Es climatizada & & \\
\hline Es al ambiente natural & & \\
\hline Es de hormigón & & \\
\hline Es de madera & & \\
\hline Es de hormigón y madera & $\mathbf{6}$ & \\
\hline Posee divisiones & & $\mathbf{6}$ \\
\hline 5.- COMERCIALIZACIÓN & & \\
\hline Consumidor final & & \\
\hline Intermediario & & \\
\hline Minorista & $\mathbf{6}$ & \\
\hline Mayorista & & \\
\hline
\end{tabular}

De las seis microempresas solo una no selecciona la materia prima la misma que es extraída de los cultivos de cada uno de los propietarios y trasladada en asno a la planta de procesamiento para extraer en jugo de caña.

En cuanto a la comercialización, los microempresarios del Sitio San Carlos del cantón Jipijapa, indicaron en un $100 \%$ que los productos derivados de la caña de azúcar lo entregan a los minoristas. Como se demuestra en los resultados de la ficha de observación existe falencia para comercializar estos productos, como son: el aguardiente, panela, alfeñique, té, punto, guarapo entre otros.

\footnotetext{
409 Vol. 5, núm. 3, julio, 2019, pp.391-414

Enrique Soledispa-Rodríguez, Carlos Artemidoro Zea Barahona, Antonio Eduardo Osejos Vásquez, Hernán Patricio Delgado Solís
} 
Los productores alegaron que el estado de las vías y la accesibilidad a las fincas afectan la comercialización de sus productos. Estos productores son los que están ubicados en las zonas que se dificulta el acceso a las fincas.

Los productores además identificaron o sugirieron una serie de problemas que de igual forma afectan este proceso de comercialización, siendo:

- Las prohibiciones para la transportación de aguardiente dificultan en gran escala su comercialización;

- La obtención del registro sanitario se ha convertido en uno de las principales problemáticas que ha frenado la comercialización en estas zonas.

- La falta de un centro de acopio que controle la calidad de los precios y funcione como entidad reguladora precios;

- Falta de un control de calidad de los productos elaborados localmente.

Todos estos aspectos constituyen factores que limitan la comercialización de las producciones locales de las mini industrias asociadas a la producción de la caña de azúcar.

Por otra parte, los resultados mostraron que más del 65\% de los productores venden sus productos localmente y de manera individual, en efectivo, directamente en la finca o en el lugar donde los procesan.

La gran mayoría de estos no posee un aliado comercial. Los microempresarios venden su producto a precios minoristas.

El proceso de comercialización trae aparejado el almacenamiento de productos, pues en este caso los fabricantes de aguardiente almacenan su producto, en tanques de 200 litros, o canecas de 20 litros.

\footnotetext{
\begin{tabular}{l|l}
\hline 410 & Vol. 5, núm. 3, julio, 2019, pp.391-414 \\
Enrique Soledispa-Rodríguez, Carlos Artemidoro Zea Barahona, Antonio Eduardo Osejos
\end{tabular} Vásquez, Hernán Patricio Delgado Solís
} 
Para obtener información del cliente sobre el consumo y la calidad de los productos derivados de la caña de azúcar se realizó una encuesta a 378 personas que representan una muestra de la Población Económicamente Activa (PEA) que está compuesta por 25100 habitantes de la misma que se obtuvieron los siguientes resultados:

E1 92\% de las personas indicaron que han consumido diversos productos de la caña de azúcar, entre ellos la panela con un $36 \%$ el alfeñique con un $26 \%$ el aguardiente con el $23 \%$ y el guarapo con un $10 \%$ y el punto con $5 \%$, como se puede observar el $85 \%$ de las personas consumen más los tres primeros productos.

Al realizar preguntas sobre algunos aspectos de calidad y consumo de los tres productos más consumidos respondieron lo siguiente:

Sobre la frecuencia de consumo de la panela el 29\% indicó que la consumen en ocasiones especiales, cada semana el 25\%, cada dos semanas el 21\% y una vez al mes $25 \%$; el consumo de azúcar sigue liderando el mercado con el 40\%, seguida de la panela con el 30\% la azúcar morena con un $26 \%$ y con el $4 \%$ endulzantes naturales, sin embargo, el $70 \%$ de los consumidores consideran que la panela es un endulzante saludable, por lo tanto el $41 \%$ lo consume por su contenido nutricional, el $30 \%$ por su calidad y $29 \%$ por otros aspectos.

\begin{tabular}{|l|r|}
\hline Opciones & Porcentaje \\
\hline Precio & $7 \%$ \\
\hline Calidad & $30 \%$ \\
\hline Disponibilidad en el mercado & $5 \%$ \\
\hline Empaque & $4 \%$ \\
\hline Facilidad de uso & $12 \%$ \\
\hline Contenido nutricional & $41 \%$ \\
\hline
\end{tabular}

\footnotetext{
411 Vol. 5, núm. 3, julio, 2019, pp.391-414

Enrique Soledispa-Rodríguez, Carlos Artemidoro Zea Barahona, Antonio Eduardo Osejos Vásquez, Hernán Patricio Delgado Solís
} 
En lo que respecta a su presentación, el 66\% indicó estar satisfecho, y el restante respondió lo siguiente:

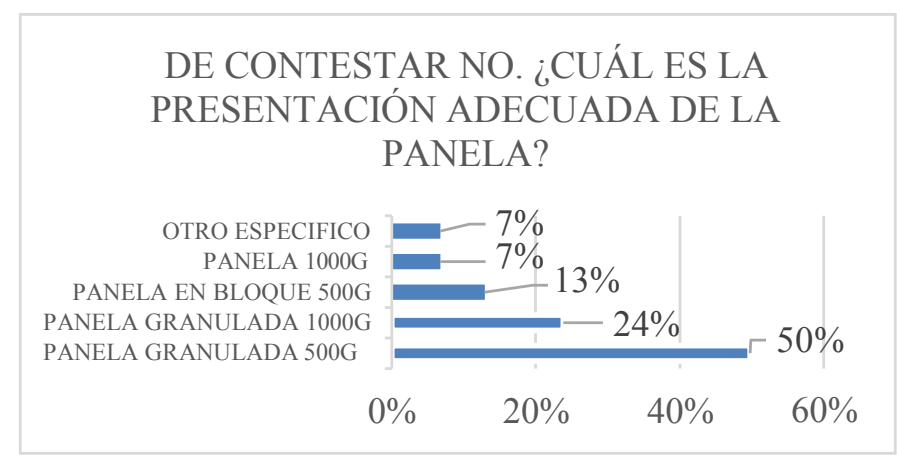

Se puede evidenciar que hay un grupo de consumidores que prefieren la panela granulada de $500 \mathrm{~g}$ la misma que en la actualidad es comercializada en diferentes locales comerciales.

Para evidenciar sobre la calidad del alfeñique se considerado cuatro opciones donde los consumidores expresaron lo siguiente:

\begin{tabular}{|l|c|}
\hline Opciones & Porcentaje \\
\hline Excelente & $8 \%$ \\
\hline Muy bueno & $27 \%$ \\
\hline Bueno & $55 \%$ \\
\hline Malo & $10 \%$ \\
\hline
\end{tabular}

Como se puede observar un pequeño porcentaje de consumidores consideran a este producto como excelente y la mayoría de ellos lo catalogan como bueno, en lo que concierne a su presentación el $47 \%$ indicó que hay que mejorarla, el $43 \%$ conforme y el 10\% está inconforme, sobre la regularidad de su consumo manifestaron lo siguiente:

\footnotetext{
412 Vol. 5, núm. 3, julio, 2019, pp.391-414

Enrique Soledispa-Rodríguez, Carlos Artemidoro Zea Barahona, Antonio Eduardo Osejos Vásquez, Hernán Patricio Delgado Solís
} 


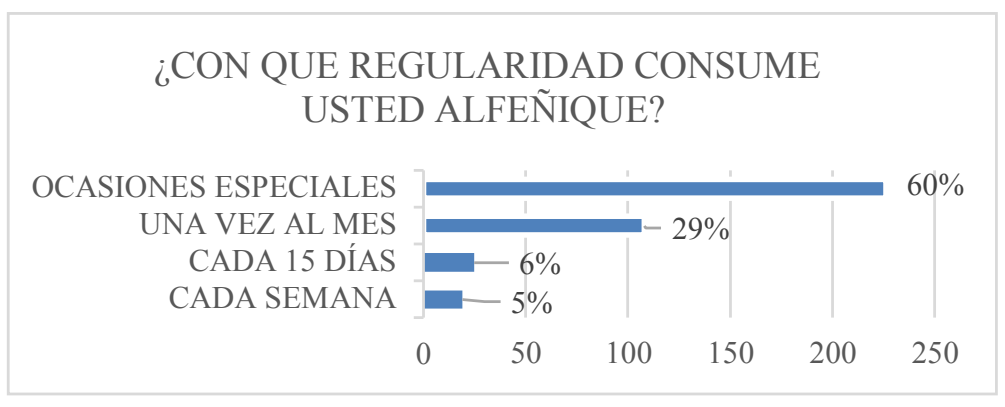

Se observa que este producto es consumido mayormente en ocasiones especiales y otro grupo de personas una vez al mes.

En lo que corresponde al aguardiente, el $61 \%$ de los consumidores indicaron que este producto es de buena calidad y 39\% restante se distribuyó entre excelente, muy bueno y malo, el 51\% de ellos también manifestó que la presentación del producto hay que mejorarla, ya que su envase no es adecuado.

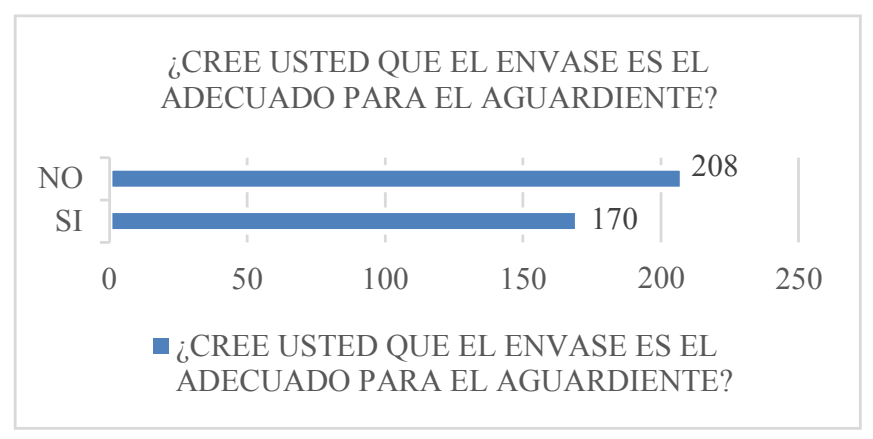

En la gráfica se puede evidenciar que un gran porcentaje de los clientes no está de acuerdo con el envase.

\section{Conclusión}

\footnotetext{
413 Vol. 5, núm. 3, julio, 2019, pp.391-414 Enrique Soledispa-Rodríguez, Carlos Artemidoro Zea Barahona, Antonio Eduardo Osejos Vásquez, Hernán Patricio Delgado Solís
} 
Las microempresas de los derivados de la caña de azúcar del sitio San Carlos no cuentan con una infraestructura adecuada para su funcionamiento de acuerdo a los parámetros de evaluación utilizados, factor fundamental para mejorar la producción y comercialización de sus productos.

Es muy notorio ver que los procesos que emplean para la producción de los diferentes derivados de la caña de azúcar son realizados en su mayoría de manera artesanal, y en algunos casos sin especificaciones técnicas.

No existen especificaciones técnicas de seguridad industrial en lo referente a la vestimenta que deben usar los trabajadores y a una señalética sobre la seguridad industrial que debe tener empresas cuando manipulan equipos que procesas materia prima para generar nuevos productos.

Los propietarios de estas empresas no llevan un sistema de control de calidad adecuado de cada uno de los productos que ellos comercializan, lo que influye en su calidad, sabor, presentación.

\section{Referencias Bibliográficas}

Gutiérrez, M. (1989). Administrar para la calidad: conceptos administrativos del control total de calidad. México: Editorial Limusa. Obtenido de https://books.google.com.ec/books?id=eVQShi8w2AUC\&printsec=frontcover\&dq=siste ma + de + control + de + calidad\&hl=es\&sa $=$ X\&ved=0ahUKEwinxY_s19zYAhUG71MKHQc qCZA4ChDoAQg3MAQ\#v=onepage\&q=sistema $\% 20 \mathrm{de} \% 20$ control $\% 20 \mathrm{de} \% 20$ calidad $\& \mathrm{f}$ $=$ false

Lester, R. H., Enrick, N. L., \& Mottley, H. E. (1989). Control de calidad y beneficio empresarial. España: Ediciones Díaz de Santos. Obtenido de https://books.google.com.ec/books?id=PSeWiHdE_6YC\&pg=PA25\&dq=sistema + de + con trol + de + calidad\&hl=es\&sa $=$ X\&ved=0ahUKEwiCnf7X19zYAhUQvlMKHY0tBhoQ6AEI $\mathrm{KzAC} \# \mathrm{v}=$ onepage $\& \mathrm{q}=$ sistema $\% 20 \mathrm{de} \% 20$ control $\% 20 \mathrm{de} \% 20$ calidad $\& \mathrm{f}=$ false

\footnotetext{
414 Vol. 5, núm. 3, julio, 2019, pp.391-414 Enrique Soledispa-Rodríguez, Carlos Artemidoro Zea Barahona, Antonio Eduardo Osejos Vásquez, Hernán Patricio Delgado Solís
} 